$\begin{array}{ll}\text { Italique } & \text { Italique } \\ \text { Poésie italienne de la Renaissance }\end{array}$

VIII | 2005

Varia

\title{
Pétrarque, la parole silencieuse
}

\section{Arnaud Tripet}

\section{(2) OpenEdition \\ Journals}

Édition électronique

URL : http://journals.openedition.org/italique/112

DOI : $10.4000 /$ italique. 112

ISSN : 1663-4438

Éditeur

Librairie Droz

Édition imprimée

Date de publication : 1 juillet 2005

Pagination : 9-25

ISBN : 2-600-01024-6

ISSN : $1423-3983$

Référence électronique

Arnaud Tripet, « Pétrarque, la parole silencieuse », Italique [En ligne], VIII | 2005, mis en ligne le 05 octobre 2009, consulté le 19 avril 2019. URL : http://journals.openedition.org/italique/112 ; DOI : 10.4000/italique.112

(c) Tous droits réservés 
A RNAUD TRIPET

P ÉTR A R Q U E,

LA PAROLE S I E N C I E S E 
à Arnaldo Pizzorusso

Et tacendo dicea (Rime, I23, I3) 


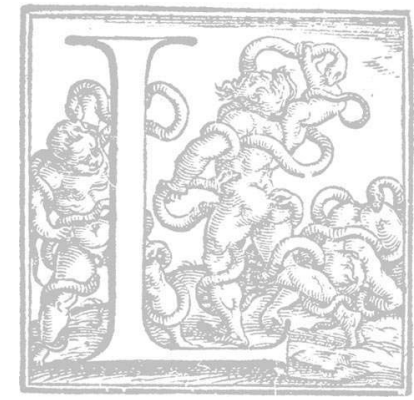

Es historiens s'accordent en général pour attribuer à Pétrarque une place inaugurale dans la culture dite bumaniste, une culture qui sacrifie avant tout aux divinités de la parole. On restitue alors celle des Anciens. On valorise le verbe en lui conférant un pouvoir inédit sur les âmes, la capacité de les convertir au bien, au vrai et au beau. Son oeuvre tant latine qu'italienne fournirait presque à l'infini des citations où la puissance bienfaisante du discours est proclamée au sein d'une épiphanie antique de la sagesse. Bientôt, et sous son influence, vont se créer en Italie, puis en Europe des écoles parallèles à celles déjà en place. Dans leurs programmes humanistes, le traditionnel trivium ne suffira plus, avec la logique, la rhétorique et la grammaire, pour qualifier les disciplines du langage en vue de la maîtrise ès arts. Va s'ajouter l'étude de l'bistoire et de la poésie, laquelle n'existait précédemment que comme une variante de la rbétorique. Le souci d'élégance expressive ira de pair avec une certaine laïcisation des contenus. Humanisme dont on dira en simplifiant outrancièrement qu'il se construit ad maiorem hominis gloriam, de l'homme «parlant» en tout cas, et tenté souvent par une copia un peu complaisante, voire incontinente.

I. Une question se pose alors: le silence va-t-il tempérer cette abondance? Va-t-il nuancer la tentation de croire que le mot a valeur ontologique, et que l'on est ce qu'on dit? Va-t-il suggérer que l'on est tout autant ce qu'on tait? Sans autre préambule, je voudrais produire deux exemples tirés de Pétrarque. Ils parlent d'eux-mêmes et le silence y prend rang avec pleine dignité dans son discours. Le premier exemple est archiconnu. Il s'agit du récit qui relate dans la lettre Familière IV, I une excursion au mont Ventoux. ${ }^{2} \dot{A}$ l'époque l'entreprise est assez originale. La montée est décrite comme harrassante. Le narrateur se plaît à le souligner, comme tout narrateur, mais aussi chez. Pétrarque pour mieux métaphoriser son expérience et lui donner une portée morale plus évidente. L'effort physique le fait penser aux difficultés du cheminement qu'impose, dans la vie de chacun, une vertu éminente. Enfin rendu, il contemple le panorama. Puis, il ouvre les Confessions de saint Augustin et tombe sur un passage du livre X qui condamne la curiosité de l'homme pour le «vain spectacle» du monde extérieur, cette libido videndi dont le lecteur pense aussitôt qu'il a bien lieu de s'accuser. Alors se détournant du paysage, il prend le chemin de la descente:

Je tournai, dit-il, les yeux de mon esprit vers l'intérieur et dès ce moment personne ne m'entendit parler jusqu'à notre arrivée dans la plaine. 
L'autre exemple est moins connu. Il figure dans une lettre, la Familière VI, 2, envoyée à un dominicain de la grande famille Colonna. Elle narre une promenade accomplie à Rome quelques années plus tôt par les deux hommes, l'un et l'autre amoureux de l'Antiquité. Les lieux traversés sont évoqués, les dialogues des deux antiquaires suggérés et la nostalgie de l'Ubi sunt retentit en présence des ruines et des espaces désolés. Puis, installé sur une éminence des Thermes de Dioclétien, Pétrarque improvise un discours sur des thèmes sans doute historiques et philosophiques. C'est ce discours que Colonna di San Vito, dans une lettre que nous n'avons pas, lui a demandé de restituer. Au terme de la sienne, Pétrarque lui répond qu'il n'en est pas capable. Comment retrouver, en l'absence de Rome, les mots inspirés par la présence de Rome? Aussi bien abandonnera-t-il au silence la profondeur exceptionnelle de ses propos d'alors. Notons que, sur les pentes du Ventoux, il abandonne au même silence toute vélleité de discours extérieur. Le contenu des deux récits est pratiquement contemporain (I336-I337). L'un et l'autre évoquent un discours exceptionnel anquel on ne peut associer que le silence. Mieux, la bauteur qui, dans les deux situations, fait se rencontrer deux versants au point presque sans espace d'une cime, héberge la parole et le silence. Ne serait-ce pas que ceux-ci se rencontrent aussi, quand l'enjeu est d'importance, en un point où le verbe comme l'espace ponctuel est et n'est pas tout à la fois?

2. La relation de la parole et du silence se retrouve dans la poésie du Canzoniere amoureux. Nous la relèverons comme le signe d'une unité de l'cuvre, au-delà des condamnations fréquentes, par son auteur même, de cette poésie d'amour.

D'abord, fait important, les traditions courtoises, stilnoviste et pétrarquienne associent souvent la femme aimée à l'idée de hauteur. On va jusqu'à voir en elle un être angélique, descendu du ciel. Le texte-culte à cet égard est le sonnet de la Vita nova: "Tanto gentile e tanto onesta pare la donna mia...». Cela peut même rendre muet, ajoute Dante: «ogne lingua deven tremando muta». Ce mutisme, les troubadours en faisaient déjà état à plus d'un titre. D'abord dans la perspective d'une psychologie ou d'une physiologie de l'amour, lequel bouleverse celui qui aime; ensuite dans la perspective d'une profondeur tout intérieure de la fin' amor. Ce qui est sûr, c'est que si la dame a un effet transformant, elle a aussi un effet paralysant. Paralysant, car on n'est jamais à sa bauteur et que ce qu'on dit est presque toujours une sorte de silence troublé par des tentatives inadéquates. Pétrarque parle de ses «roche rime», pauvres (scarse) pour ne pas dire nulles. «Allor dirà che mie rime son mute» (Rime, 248, I2). Mais voilà un silence qui ne conduit pas tout à fait au mutisme. C'est un silence inclus, qui a la force d'une complémentarité dialectique et qui fait 
alliance avec le langage. Il en résulte d'ailleurs un véritable traitement thématique et un débat constamment repris entre le dire et le non-dire.

Si ineffable qu'en soit l'objet, on n'échappe pas au devoir de le célébrer. On ne résiste pas non plus au désir de soulager un ccur à ce point sollicité. Les larmes et les soupirs appartiennent dans le Canzoniere à ce régime d'un soulagement irrésistible et bienfaisant, que le poète traduit très fréquemment par le verbe sfogare ou sfogarsi. Enfin, surtout peut-être, chanter a valeur de destin et le poiétès ne peut pas ne pas s'adonner à sa pulsion fabricatrice. N'est-il pas appelé à mettre au monde des objets impeccables et à percer les secrets de la versification, à multiplier antithèses et paradoxes, à jouer comme le musicien des rythmes et des variations, à remettre ses pieds dans les pas des Anciens, des troubadours, des Toscans pour produire ses propres choréograpbies? Nous ne saurions oublier le dictamen du talent, le goût de l'exploit, les satisfactions de l'ingéniosité. C'est entre autres ce que la poésie européenne ne cesse de nous rappeler quand elle pétrarquise à tort ou à raison, ou quand elle refuse de le faire au nom du naturel expressif on existentiel.

Or, si la parole est thématisée dans l'cuvre du grand «trecentista», le non-dire l'est également. Il l'est de quatre manières en tout cas, et nous allons y jeter un coup d'oeil si vous le voulez bien. Je veux parler d'une pratique de la sévérité, de la prétérition, de l'bypothèse et de la correction.

a) Le jugement sévère est souvent à l'oeuvre dans les textes de Pétrarque, surtout quand il parle de sa propre poésie ou de sa personne. Fausse ou vraie modestie? La question n'est pas là, car ce qui compte ici c'est au nom de quoi ou en vertu de quelle allégeance louable se font ces déclarations dénigrantes. Or, il va presque sans dire que le jugement fonctionne alors chez Pétrarque comme l'attestation d'une sagesse qui prend ses distances. Le premier sonnet du Canzoniere, qui est une palinodie du recueil, propose l'image d'un bomme mûri et assagi dans sa conception du monde. Il veut que l'on sache que tout est vain ici-bas, particulièrement les émotions incontrôlées de la folie amoureuse. Il veut que l'on sache qu'il le pense. Ses juvenilia trabissaient des faiblesses qu'il regrette, ce qui ne l'empêche pas de les retravailler maintenant encore, de les polir et de les commenter en latin dans les marges de son manuscrit jusqu'an seuil de la mort. ${ }^{3}$ Sévérité valorisante, donc. Elle voudrait faire taire ce qui ne mérite pas d'être trop revendiqué. Mais ce silence est produit par des mots qui ne laissent pas d'être favorables à l'image de celui qui tient la plume maintenant.

b) Outre cette sévérité qui «rejette» avantageusement, signalons une autre forme du non-dire que l'on appelle prétérition. Cette fleur de rhétorique émaille les plates-bandes pétrarquiennes. Elle consiste à déclarer qu'on refuse de s'exprimer sur un sujet donné. C'est souvent un détour pour s'exprimer 
justement. "Je ne te dirai, je ne peux pas te dire... ceci, cela, et en particulier, combien je t'aime...». Jeu de cache-cache, manière détournée de dire en faisant semblant de ne pas dire. Comme la litote, c'est un procédé qui «étoffe» sous des apparences discrètes. Il laisse deviner que ce dont il s'agit a une importance particulière, il avoue une intensité qui entrave sa propre expression. En réalité, dans un tel contexte, la prétérition a une forte connotation byperbolique. Mon amour est trop grand pour que je puisse l'exprimer, d'abord parce qu'il entame mes ressources expressives, ensuite parce que son objet dépasse tout ce qu'on pourrait en dire. Ses soupirs, mot-clef chez Pétrarque, ne trouvent pas d'issue dans les mots:

I miei gravi sospir' non vanno in rime

(Rime, 332, I I)

La souffrance amoureuse pèse tant qu'elle ne parvient pas à s'engager dans la musique ou la danse des mots. Je reste sidéré devant l'objet qui m'éblouit. On se doute bien que cette incapacité avonée de dire dit beaucoup. Elle déclenche l'byperbole de l'éloge et une soumission totale à la domna, pour parler occitan.

c) La prétérition de l'impuissance s'ouvre sur l'hypothèse d'un chant qui serait adéquat. Les conséquences en seraient symétriquement byperboliques. Au «je ne peux pas» de la prétérition s'ajoute un «si je pouvais...». Nous pourrions appeler orphique la physionomie de ces hypothèses où la voix de Pétrarque serait à la hauteur. Car, s'il est admis et répété que la voix de Laure bouleverse l'ordre naturel, celle de Pétrarque, au diapason du ressenti, ferait pleurer d'émoi les rochers même:

avrei fatto parlando

romper le pietre, et pianger di dolcezza

$$
\text { (Rime, 304, I3-14) }
$$

Les ours et les tigres ne résisteraient pas davantage à la parole de Laure, si je savais la reproduire. Sa simple présence dans le paysage régénère la nature, déclenche un amour universel comparable au tropisme des créatures pour leur Créateur, selon la pensée thomiste. Elle est un miracle à la fois crée et créateur. Parfois, l'amant a lui-même l'impression que ses déclarations devraient troubler Laure. Il sait et répète qu'en principe la poésie possède un pouvoir inégalable :

Nulla al mondo è che non possano i versi

(Rime, 239, 28) 
Ces affirmations confiantes relèvent certes d'un credo inébranlable. On le retrouve dans les pages de l'bumaniste qui ne cessent de réitérer leur foi dans le verbe. Mais ce n'est pas à cela que nous voulons être attentifs aujourd'bui. Il nous importe de remarquer plutôt que le plus souvent la poésie de Pétrarque mêle au principe de sa toute-puissance mille nuances et mille degrés. Ces doutes parlent souvent en faveur de Laure et de son pouvoir sur l'amant. Ils parlent, voilà justement ce qu'il convient de relever en l'occurrence.

d) Au silence soubaité dans le jugement sévère porté par le poète sur son oeuvre amoureuse, à l'effacement éloquent de la prétérition et de l'bypothèse hyperbolique, ajoutons encore la figure de la correction qui, de manière voisine, affirme en niant. Plus précisément, elle tire explicitement le mieux du moins bien. Celui-ci n'est donc pas effacé et le moins devient ainsi le tremplin d'un progrès, une sorte de faute positive, une felix culpa. L'écrivain a peut-être l'impression qu'il s'approche alors du vrai, du bien et, pourquoi pas? de Laure et même de Dieu. Voici, par exemple, un texte à plusieurs étages dont chacun précise ou corrige le précédent. D'abord, l'amant affirme que Laure est le guide de sa vie, mais, qu'étant morte, il est obligé de l'imaginer (premier étage), elle qui, en fait, est sous terre (deuxième étage), ou mieux (troisième étage), au ciel:

$$
\begin{aligned}
& \text { Imaginata guida la [la mia vita] conduce } \\
& \text { ché la vera è sotterra, anzi nel cielo }
\end{aligned}
$$

(Rime, 277, 9-10)

Que de degrés dans l'identité de cette nouvelle Béatrice! On remarquera surtout que ce qu'on rejette subsiste et que, dans ce dépassement, on ne laisse pas de prêter l'oreille à l'état précédent. Le silence, dans ce procédé - comme dans ceux que nous avons déjà signalés - est totalement mêlé, voire complice, d'un aboutissement dynamique de l'expressivité verbale.

3. Dans son existence aussi, l'homme de la parole juste cherche des conditions favorables à son surgissement. Et ce surgissement est programmé bien souvent avec l'aide d'un silence ambiant. Il s'agira par exemple de ne rien dire pour mieux se dire intérieurement ce qu'il faut se dire. Il s'agira aussi de ne rien vouloir des rumeurs de l'extérieur. En descendant le Ventoux, le promeneur va jusqu'à se fermer aux questions de son frère qu'inquiète un mutisme si visiblement délibéré. La solitude que Francesco recherche dans la nature correspond bien pour lui à l'extinction soubaitée des voix qui pourraient troubler la sienne, ce sentiment intérieur qu'il faut préserver de tout parasitage. En bon disciple de saint Augustin, il est encouragé à penser que c'est au fond de nous-mêmes que se trouve ce qui mérite d'être cru et dit, et qu'on n'y accède pas sans quelques mesures propédeutiques. Les stoïciens aussi parlaient dans 
le même sens d'un discours intérieur, d'un «endiathétos logos» dont l'écoute nécessite le rejet de tout bronillage. Chez Pétrarque et chez tant d'autres ce discours enveloppé dans le silence se développera en une méditation plus ou moins rêveuse. Dans les solitudes de Capranica ce sera un travail de la mémoire et de l'imagination en vue de l'épopée de Scipion qui incube en lui (Familières, II, I2). Il se rend à Rome, mais les troubles où la ville est plongée le maintiennent en quarantaine à bonne distance. Les bandes rivales des Orsini et des Colonna font cliqueter leurs armes et, au grand étonnement de tous, le promeneur qu'il est n'y prête pas attention, plongé dit-il dans son univers intérieur, celui sans doute de l'Africa et du De viris illustribus. Même insouciance des bruits menaçants dans la traversée de la forêt d'Ardenne, car la solitude se prête pour lui au seul chant qui s'élève dans son coeur à la pensée de Laure absente (Rime, I76).

La promenade est bien souvent un déplacement, ou un replacement intérieur cherchant la rencontre mentale avec ce que le promeneur aime, avec ce qui compte pour lui. La relation est étroite alors entre ce mouvement extérieur toujours plus solitaire de la déambulation et un mouvement intérieur toujours plus exclusif. Le physique et le psychique se répondent même à tel point que l'ascension de la montagne et des pensées sont mises syntaxiquement sur le même plan: "De pensée en pensée, de montagne en montagne / me guide Amour...»:

Di pensier in pensier, di monte in monte mi guida Amor ...

(Rime, I 29, I-2)

Certes, l'Amour qui mène la marche semble un personnage extérieur. Mais il est bien évident que la personnification du sentiment n'ôte rien à la localisation tout intérieure de celui-ci. On lit souvent dans la poésie stilnoviste que les mots écrits par le poète sont le résultat d'une dictée de l'Amour, et Pétrarque de reprendre:

Amor par ch' a l'orecchie mi favelle

(Rime, 21 8, 5)

En fait, la dictée d'Amour, dans cette doctrine, dit l'authenticité du sentiment, elle atteste ce qui se passe dans le profond du coeur. Dante se définit lui-même en ces termes au Purgatoire (XXIV, 52-54):

... I' mi son un che quando

Amor mi spira, noto, e a quel modo

ch' e' ditta dentro, vo significando. 
L'amour ressenti est souvent aussi un lieu, un lieu sanvage qui accueille le surcroît affectif et le chant d'un cceur qui se met au diapason des oiseaux, du vent et de l'eau courante.

Mais c'est aussi l'endroit où Laure est apparue dans toute sa beauté et où il entend la retrouver en pensée, voire en réalité, quand il s'en est éloigné. Souvenirs plus ou moins fictifs, constats enchantés d'hallucinations, mise en scène dans un avenir hypothétique où Pétrarque imagine qu'il est l'objet d'une émotion funèbre de la part de Laure, le lieu déborde de modélisations intérieures, dans ce vallon de Vaucluse! C'est bien la poésie du pélerinage constamment repris, poésie d'un être qui voyage dans le secret de sa tête autant que sur la terre! Or c'est encore le silence des lieux qui fait ressurgir cette histoire plus ou moins rêvée. Une sorte de fétichisme des choses qui ont effleuré et pourraient encore effleurer celle qui trône au milieu d'elles occasionne ce qu'on a appelé un renouvellement du coup de foudre et jusque sur la tombe de Laure. Mais alors, il se plaint à la mort d'une intrusion qui transforme en un pélerinage désespérant les rêveries du lieu où il introduisait l'aimée. ${ }^{4}$ Le discours intérieur devra établir son assise dans le ciel. Pour le survivant, la vie est devenue un silence terrestre. Elle est, comme il dit, une histoire achevée, un récit, une favola, une parole qui ont été interrompus:

La mia favola breve è già compita

(Rime, 254, I 3)

4. Mais on sait que tout ne finit pas là et qu'après la $265^{e}$ pièce cent nouveaux poèmes, que les éditeurs ont rangés sous le titre de In morte di Madonna Laura, vont prendre la succession de ce qui avait été écrit In vita di Madonna Laura. Le silence de la mort n'en sera donc pas un, ni sur le plan quantitatif, ni surtout qualitatif, ce dernier point n'ayant pas été suffisamment reconnu, à mon sens, par la critique pétrarquienne. Cette riche bécatombe poétique qui conduit à une canzone à la Vierge, prolongement ou substitution de Laure, mériterait donc un examen soutenu.

Évidemment, le deuil commence par se déclarer inexprimable:

Qual ingegno a parole

poria aguagliare il mio doglioso stato?

(Rime, 268, 18-19)

La perte est sans égale et l'amant sent que son coeur lui appartient de moins en moins. Il a gagné le ciel, non sans ressentir encore une totale déréliction. Comment peut-il battre encore?

Madonna è morta ed ha seco il mio cuore

(Rime, 268, 4) 
Seule l'image de l'errance rendra compte d'une vie qui a perdu son étoile, vainement recherchée, «lei cercando invano». On peut bien penser que les ressources rbétoriques auxquelles nous avons fait allusion sont réutilisées dans un contexte à ce point mystérieux et byperbolique. Encore et toujours, un silence éloquent se met au service de l'extrême, d'un extrême qui est maintenant un absolu de silence.

Et pourtant, les lecteurs des Rime le savent bien, c'est dans la mort même que la voix de Laure se fait le plus entendre. On nous avait dit à maintes reprises que ses paroles possédaient une douceur invincible, capable d'ennoblir les plus grossières étoffes. Pétrarque le redit au passé dans In morte di Madonna:

Oimè il parlar ch'ogni aspro ingegno et fero

facevi humile, ed ogni huom vil gagliardo!

(Rime, 267, 3-4)

Seulement, jusqu'à présent, la situation locutoire de Laure restait générale: contexte rare, contenu caché, à part quelques salutations et un ou deux adieux. Or, paradoxalement, la grande distance de la mort suscite une intimité inédite, des propos rapportés, des dialogues. Oui, le grand silence libère la parole. Un lien nouvean se tisse qui dérive d'une distance posée et abolie. L'ambiguité ou la complexité existaient avant la mort de Laure. Celle-ci suivait l'injonction courtoise de la femme qui se refuse sans que cela éteignit, tout au contraire, l'ardeur de l'amant. Déjà elle était présente et absente ou mieux, chose ressentie autant que vue, ou vue comme un mirage:

I' l'ò più volte (or chi fia che m'il creda?) ne l'acqua chiara et sopra l'erba verde veduto viva ...

(Rime, I 29, 40-42)

Maintenant l'éloignement et l'intimité s'accentuent conjointement. Laure descend du ciel, encourage le malheureux survivant, lui dit de ne pas pleurer puisqu'elle a trouvé la béatitude, petite ellipse qui laisse dans l'ombre la vraie cause des pleurs, la perte éprouvée par l'amant. Une bonne vingtaine de pièces font apparaître Laure dans des conduites d'accueil, de sollicitude, de compassion à l'égard de son compagnon désemparé. Elle se soucie de son salut, l'associe à une éternité commune. Deux fois (302, 362), mû par une force mystérieuse, il s'envole jusqu'à elle. Plus souvent, c'est elle qui descend jusqu'à lui, de nuit surtout, et près de l'aube quand les rêves disent vrai. Alors qu'il n'était pas sûr auparavant qu'elle écoutât ni surtout aimât ses plaintes, il s'aperçoit maintenant que ce qu'il lui dit de ses souffrances fait monter les 
larmes à ses yeux. Dans l'admirable sonnet 356, cette situation, ò̀ enfin les choses peuvent être dites, rappelle un peu l'avant-dernier chapitre de l'Éducation sentimentale où Mme Arnoux fait enfin comprendre à Frédéric combien son cour à elle avait battu à l'unisson du sien. Croce a rapproché ce chapitre du roman flaubertien, qui commence par «Il voyagea», de trois autres sonnets (3I5-3I7) où Pétrarque déplore que la mort ait empêché une évolution de ses rapports avec Laure. Il aurait tant soubaité l'avènement d'un âge où les dangers d'éros sont moins vifs et auraient autorisé de douces confidences. Cela reste une bypothèse irréelle in vita di Madonna. Il faut croire que la vraie combinaison de l'intimité et de la distance n'est réalisable chez. Pétrarque que dans une relation entre une morte - un ange - et un vivant, comme plus tard dans la Spirite de Gautier ou déjà dans le Paradiso dantesque? Dans le sonnet 356 post mortem, que nous avons mentionné, le caractère inéluctable de la relation entre silence et parole se traduit par une touche où le dire et le non-dire sont dotés d'une force égale. L'amant ayant raconté à Laure son parcours et ses souffrances, il est écrit: "elle se tait» (Ella si tace)... Quel silence! N'exprime-t-il pas autant, voire plus, qu'aucune parole?

I' incomincio da quel guardo amoroso che fu principio a sì lungo tormento, poi seguo come misero et contento, di dì in dì, d'ora in hora Amor m'ha roso.

Ella si tace, et di pietà depinta fiso mira pur me; parte sospira et di lagrime honeste il viso adorna

(Rime, 356, 5-I I)

Malheureusement la distance a trop souvent le dessus. Les apparitions disparaissent à l'arrivée du jour. Pourtant, elles reviennent et maintiennent dans un rythme alterné présence et absence, pleurs et consolation, silence et parole. Tout comme l'espace qui postule l'ici et l'ailleurs, le temps appartient au multiple et n'est pas immuable. Seul le paradis assurera l'unité permanente d'un êtreensemble. Pour l'beure, la recherche d'une union mystique s'attarde volontiers à la médiation de Laure. Oui, l'élan vers le haut a tendance à stationner aux pieds d'une fermme céleste, angélique, une femme envoyée et entraînante. Elle répìte un peu la scène de l'Annonciation, mais c'est la figure mariale qui vient éclairer un ange quelque peu déchu et qui ne peut s'empêcher de féminiser Dieu ou de diviniser la femme, avant de se repentir d'avoir pu le faire. Pétrarque emprunte alors pour la millième fois la voie de la réorientation (appelons-la la correction). Peut-être que le mot paix (pace) qui clôt la prière finale à la Vierge dit bien que le monde de l'alternance antagoniste ou complémentaire, 
peu importe, du langage et du silence, connaît alors l'aspiration d'un apaisement et d'une élimination douce et globale, le tout au terme d'une série de médiations (mère-Fils-Dieu) dont la paix serait l'achèvement:

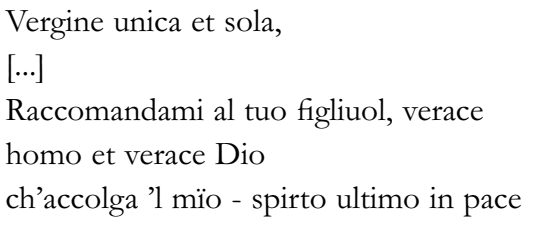

(Rime, 366, I33-1 37)

5. Sans prétendre conclure, sinon par mon propre silence, je terminerai rapidement en rassemblant sur le terrain qui a été le nôtre quelques éléments de confirmation. On remarquera d'abord que la grandeur unique dont Pétrarque entend doter la fermme aimée est le résultat d'une addition de mérites que sa poésie se plaît à énumérer, poésie d'éloge, poésie immortalisante. Mais c'est le résultat aussi d'une soustraction en ce sens que Laure pour être unique doit être déponillée de ce qui la ferait ressembler au commun des mortels. Sa différence est perceptible comme la conséquence d'une purification. Elle condamne par sa simple essence le vacarme et la vulgarité du monde. Elle s'avance non pas tellement masquée, ce sont les autres qui le sont, mais voilée. Son sourire est à la fois mystère et évidence. Cette sélection qui définit la nature de la figure centrale des Rime rencontre évidemment une pratique de la poésie où le non-dit est une beureuse exploitation du silence, lequel ne se contente pas de border les mots ou de favoriser la musicalité rythmique du discours. La figure de la correction semble travailler explicitement à cette victoire d'un essentiel qui, comme chez. le sculpteur ou l'alchimiste, contraint le poète à ôter toute matérialité encombrante sur le chemin d'une perfection presque indicible. "Je n'ai crée mon oewvre que par élimination», écrit Mallarmé. C'est dire que, si on le suit, le maximum est aussi un minimum implicite, un simple souffle où la vie dans sa définition hébraïque de Ruach s'exprime: minimum expressif qui est un maximum ontologique. Dieu même se révèle ainsi à Élie après avoir écarté d'autres manifestions naturelles plus tapageuses:

Et après le feu, un murmure doux et léger. Quand Élie l'entendit, il s'enveloppa le visage de son manteau, il sortit et se tint à l'entrée de la caverne. (I Rois, I 2-I 3)

Plus près de nous, Paul Celan a repris à son compte cette image hébräque du souffle. Il parlait à propos de sa poésie de Atemreflexen. ${ }^{6}$

Depuis Pétrarque la route est donc longue et riche où s'illustrent de telles conceptions. Ce serait là l'objet de tout un travail à entreprendre que je n'ai 
même pas le temps d'effleurer. Après Mallarmé et Celan, je soumets seulement à votre réflexion deux noms pour amorcer l'idée d'une continuité occidentale, et non seulement bouddhiste ou taoiste, dans cette dialectique féconde de la parole et du silence: les noms de Leopardi et de Verlaine. Le premier pose comme poétique et euphorique un au-delà éprouvé dans les mots qui sont vagues et possèdent ainsi un mutisme parlant. Indépendamment des mots eux-mêmes, il déclare encore, toujours dans son Zibaldone, que les choses cachées possèdent ce même caractère, car elles suscitent un mouvement vers l'infini où la conscience s'abandonne à une sorte de naufrage délicieux. "E il naufragar m'è dolce in questo mare», dit-il, on s'en souvient, dans l'admirable Infinito.

L'Art poétique de Verlaine propose également une exploitation du non-dit dans le dit. Au-delà même de l'irrégularité prosodique, il exprime sa préférence pour une sorte de méprise sémantique. Tous ces effacements libèrent l'âme sur les chemins de ce qu'il appelle la «bonne aventure». Vrai combat poétique contre le despotisme paralysant du sens et des formes, ce dépouillement comme dans les autres exemples évoqués, et notamment Pétrarque, est gage de richesse. Certes, il ne faut pas tout mélanger et oublier que chaque expérience poétique ne renvoie qu'à elle-même et à son temps. Il n'empêche que pour notre poète du $X I V^{e}$ siècle ces éclairages, tout indirects qu'ils sont, ne s'en révèlent pas moins précieux. Et cela même si l'on garde en mémoire que cet homme de I304 appartient encore à la culture courtoise qui prône le secret pour des raisons d'abord sociologiques - la dame était mariée - et ensuite pour des raisons qui relèvent d'une psychologie de l'intériorité, comme nous l'avons déjà suggéré. Depuis la fin du XIII e siècle et avec le dolce stil novo en particulier, l'érotique courtoise tend de plus en plus à se spiritualiser, autant dire à se sublimer. C'est enfin un éros en anima, pour parler comme Jung, où les qualités féminines sont poussées jusqu'à leur extrême avec la pudeur qui cache, l'bumilité qui voile, la parole qui enchante et élève par sa douceur et qui se propage dans un climat de retenue. Il me semble surtout qu'à travers cette expérience de la relation à l'autre et à soi-même qu'est le Canzoniere, Pétrarque met en oenvre ou découvre ce que j'appellerai un deuxième temps de la poésie, le deuxième temps de toute poésie authentique, fût-ce à l'égard d'elle-même. Verlaine disait «Et tout le reste est littérature», Pascal «La vraie éloquence se moque de l'éloquences. Le véritable triomphe du mot, c'est un triomphe sur le mot, c'est l'accession au souffle, invoqué comme réconfort auprès de Laure et métaphorisé dans les soupirs amoureux qui disent l'ardeur et son exténuation. C'est cette douce retombée que Pétrarque appelle le souffle et qu'il associe, Dieu aidant, à la paix («il mio spirto ultimo in pace»).

On sait le combat moral mené contre sa faiblesse et les contrariétés dans l'oeuvre latine surtout. On connait l'ingéniosité de cet artiste des mots dans les infinies variations d'éros qui pewvent lasser jusque dans leur réussite. Ce 
Arnaud Tripet

combat et cette ingéniosité en acte n'en sont pas moins nécessaires dans le destin verbal de Pétrarque. Ils appartiennent à la dialectique qui conduit à tout moment de sa trajectoire à ce souffle où la poésie fait se rencontrer l'esthétique et l'ontologique, souffle de Laure, souffle de Dieu, souffle du poète.

Arnaud Tripet 
Pétrarque, la parole silencieuse

I. E. Garin, L'Éducation de l'homme moderne (I400-1600), trad. fr., Paris, I 968.

2. A. Tripet, Pétrarque ou la connaissance de soi, Paris, 2004, p. 57-70.

3. G. Contini, Saggio di un commento alle correzioni del Petrarca volgare, Firenze, I947; F. Chiappelli, «Non satis triste principium», Pétrarque et le pétrarquisme, Versants, 7 (I985), p. 3-I4.

4. L. G. Gros, «A miracol mostrare», Accents modernes de Pétrarque, dans «Cahiers du Sud», 320 (1953), p. 35.

5. B. Croce, «Il sogno d'amore sopravvivente alla passione», dans Poesia antica e moderna, Bari, I 950 (3e édition), p. I62 suiv.

6. Non sans évoquer Pétrarque dans Die Niemandsrose en relation avec les thèmes de l'exil et de l' «abîme» de Babel:

(Und wir sang die Warschowjanka

Mit geschliften Lippen, Petrarca.

In Tundra-Ohren, Petrarca.)

Und es steigt eine Erde herauf, die unsre

diese.

Und wir schicken

keinen der Unsern hinunter

zu dir,

Babel. 